\title{
AN APPROXIMATE EXPRESSION FOR THE VALUE OF AN ASSURANCE, LIFE AGAINST LIFE.
}

To the Elitor of the Assurance Magazine.

SIR,-The following short investigation may, perhaps, be useful to some of your readers. It will be observed that the resulting formula is much more simple than the ordinary one for the single premium for a contingent assurance; and it has been found to be sufficiently accurate for practical purposes.

\section{Problem.}

To find the present value of $E 1$ to be received at the end of the year in which a life aged $x$ may fail, provided that such event happen during the lifetime of another, aged $y$; the chance of both dying in the same year being neglected.

Let $S=$ value required;

$$
\text { then } \begin{aligned}
\mathrm{S} & =\Sigma v^{n}\left(p_{x, n-1}-p_{x, n}\right) p_{y, n} \\
& =\Sigma v^{n}\left(p_{x, n-1} p_{y, n}-p_{x y, n}\right) ;
\end{aligned}
$$

but $\Sigma v^{n} p_{x y, n}=a_{x y}$

and $\Sigma v^{n} p_{x, n-1} p_{y_{n} n}=\frac{l_{x} l_{y+1}}{l_{x^{*}} l_{y}} v+\frac{l_{x+1} \cdot l_{y+2}}{l_{x} l_{y}} v^{2}+\frac{l_{x+2} l_{y+3}}{l_{x^{2}} l_{y}} v^{3}+\& c$.

$$
\begin{aligned}
& =\frac{l_{y+1}}{l_{y}} v\left(1+\frac{l_{x+1} \cdot l_{y+2}}{l_{x} \cdot l_{y+1}} v+\frac{l_{x+2} \cdot l_{y+3}}{l_{x} \cdot l_{y+1}} v^{2}+\& \mathrm{e}\right) \\
& =v p_{\mathrm{y}}\left(1+a_{x, y+1}\right) \\
& \therefore \mathrm{S}=v p_{y}\left(1+a_{x, y+1}\right)-a_{x y *} \\
& \text { I am, Sir, } \\
& \text { Yours truly, }
\end{aligned}
$$

